\title{
PRISM como predictor de mortalidad en la unidad de cuidados intensivos pediátricos del Instituto Nacional de Salud del Niño, Perú 2012
}

\section{PRISM as predictor of mortality in pediatric intensive care unit of the National Institute of Child Health, Peru 2012}

Correspondencia Raffo Escalante-Kanashiro MD raffo.escalante@gmail.com

Recibido: 15/03/2016

Arbitrado por pares

Aprobado: 27/04/2016

\section{Citar como:}

Vásquez $A$, Herrera-Perez $E$

Tantaleán J, Escalante-Kanashiro R. PRISM como predictor de mortalidad en la unidad de cuidados intensivos pediátricos del Instituto Nacional de Salud del Niño, Perú 2012. Acta Med Peru. 2016;33(1):9-14

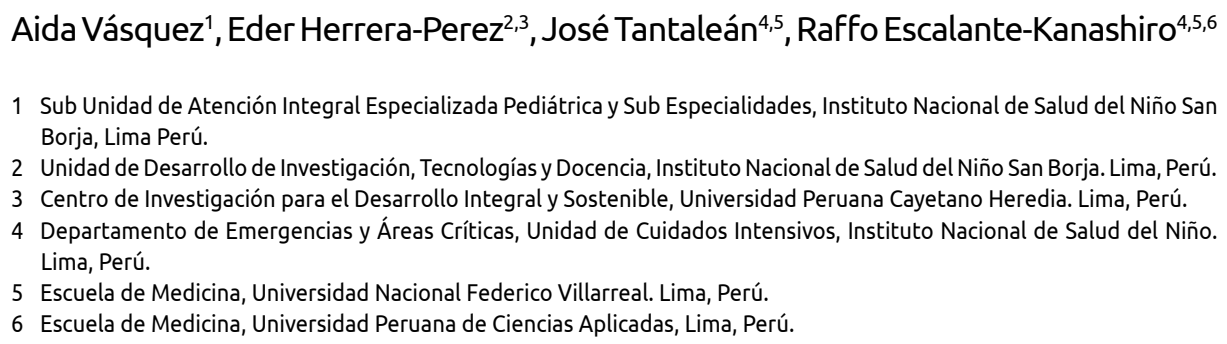

1 Sub Unidad de Atención Integral Especializada Pediátrica y Sub Especialidades, Instituto Nacional de Salud del Niño San Borja, Lima Perú.

2 Unidad de Desarrollo de Investigación, Tecnologías y Docencia, Instituto Nacional de Salud del Niño San Borja. Lima, Perú.

3 Centro de Investigación para el Desarrollo Integral y Sostenible, Universidad Peruana Cayetano Heredia. Lima, Perú.

4 Departamento de Emergencias y Áreas Críticas, Unidad de Cuidados Intensivos, Instituto Nacional de Salud del Niño. Lima, Perú.

5 Escuela de Medicina, Universidad Nacional Federico Villarreal. Lima, Perú.

6 Escuela de Medicina, Universidad Peruana de Ciencias Aplicadas, Lima, Perú.

\section{RESUMEN}

Introducción: La escala de PRISM es un predictor de mortalidad pediátrico que considera el riesgo de morir a partir del grado de afectación de 14 parámetros fisiológicos. Éste ha sido aplicado en diferentes partes del mundo, mostrando algunas discrepancias en su poder predictivo. Objetivos: Evaluar el rendimiento del escore PRISM como predictor de mortalidad de los pacientes hospitalizados en la Unidad de Cuidados Intensivos Pediátricos (UCIP) del Instituto Nacional de Salud del Niño (INSN) en Lima, Perú. Material y Método: Se analizó la base de datos de pacientes ingresados a la UCIP-INSN durante el año 2012. Se calculó la curva ROC como medida de capacidad discriminativa. La calibración fue evaluada con la prueba de Hosmer y Lemeshow. También se calculó la tasa de mortalidad estandarizada (TME). Resultados: De un total de 388 casos el 17,3\% fallecieron. Según escore de PRISM, el 91,1\% tuvo un "bajo riesgo", el 7,1\% un "moderado riesgo" y el $1,8 \%$ "alto riesgo". Se encontró asociación entre las categorías del PRISM y mortalidad ( $p<0,001)$. La curva ROC para PRISM fue 0,73 (IC95\%: 0,66-0,80), la TME fue 1,01 y la calibración fue buena $(p=0,65)$. Conclusiones: El escore PRISM muestra un rendimiento adecuado como predictor de mortalidad.

Palabras clave:

Unidades de Cuidado Intensivo Pediátrico; Predicción; Mortalidad (fuente: DeCS BIREME). 


\begin{abstract}
Introduction: The PRISM scale is a predictor of mortality in pediatric patients which considers the risk for dying by assessing 14 physiological parameters. The scale has been used in different parts of the world, showing some discrepancies in its predictive power. Objectives: To assess the performance of the PRISM score as a predictor of mortality in patients hospitalized in the Pediatric Intensive Care Unit (PICU) of the Instituto Nacional de Salud del Niño (INSN) in Lima, Peru. Material and methods: We analyzed the database of all patients admitted to the PICU in the INSN during 2012. We calculated the ROC curve as a measurement for discriminative capacity. The calibration was assessed with Hosmer and Lemeshow test. We also calculated the standardized mortality rate (SMR). Results: Out of 388 patients who were admitted, $17.3 \%$ died. According to the PRISM score, $91.1 \%$ had "low risk", $7.1 \%$ had "moderate risk", and $1.8 \%$ had "high risk". We found an association between the risk categories in the PRISM scale and mortality $(p<0.001)$. The ROC curve for the PRISM scale was 0.73 ( $95 \%$ IC: $0.66-0.80$ ), the SMR was 1.01, and there was a good calibration $(p=0.65)$. Conclusions: The PRISM score shows adequate performance as a predictor for mortality.
\end{abstract}

Key words:

Intensive Care Units, Pediatric; Forecasting; Mortality (source: MeSH NLM).

\section{INTRODUCCIÓN}

En la práctica clínica diaria existe la preocupación constante de cómo clasificar a los pacientes para su mejor tratamiento. La valoración de la gravedad así como la inestabilidad clínica y pronóstico es un componente vital a enfrentar en la unidad de cuidados intensivos pediátricos (UCIP), requiriendo de una evaluación eficaz y continua en pacientes en estado crítico [1,2].

Los puntajes de predicción de mortalidad constituyen una herramienta útil que permiten comparar la mortalidad observada en una población con la estimada por el modelo de predicción. Dichas escalas realizadas en forma rutinaria, nos permiten prever de manera más precoz la mortalidad, mejorar la calidad de atención, fortalecer la capacitación de los médicos y además clasificar de manera más eficaz a los pacientes instaurando un tratamiento precoz y adecuado, permitiendo además corregir errores [2-5].

El escore Pediatric Risk of Mortality (PRISM), es el sistema de valoración pediátrico más utilizado [6] y muestra un buen rendimiento predictivo de mortalidad $[7,8]$ que es independiente de la institución en la que se aplica [9]. Por otro lado el rendimiento del PRISM puede no ser el mismo en todas las poblaciones debido al diferente patrón de enfermedades que la afectan o características particulares de dicha población [10]. Este estudio evaluó el rendimiento del escore PRISM como predictor de mortalidad en los pacientes hospitalizados en la UCIP del Instituto Nacional de Salud del Niño (INSN) durante año 2012

El Instituto Nacional de Salud del Niño es una institución de alta especialización dentro del sistema de salud y es referencial en atención de salud pediátrica en el Perú.

\section{MATERIAL Y MÉTODOS}

Estudio cohorte retrospectiva con todos los pacientes ingresados a la UCIP entre el 1 de Enero del 2012 al 31 de Diciembre del 2012 en el Instituto Nacional de Salud del Niño, centro de referencia nacional del Ministerio de Salud del Perú para pacientes pediátricos ubicado en Lima, Perú. La UCIP cuenta con 23 camas, un tiempo promedio de 12 días de estancia. El estudio fue aprobado por el Comité de Ética del Instituto Nacional de Salud del Niño.

Se incluyó a pacientes entre 1 mes de edad y 16 años, de ambos géneros, que tengan más de 24 horas de hospitalización y con los datos completos para calcular el score de riesgo. De un total de 400 pacientes, 12 tenían menos de 24 horas y/o no tenían datos completos en la historia clínica, por lo cual fueron excluidos del estudio, quedando 388 pacientes.

EI PRISM [11] fue calculado al momento de ingreso del paciente a partir de 14 indicadores fisiológicos que se obtienen del manejo rutinario de los pacientes (Tabla 1 ), y según los valores obtenidos se les asigna un puntaje que sumado va de 0 a 76 y se estratifica de bajo riesgo si es $<20$ puntos, moderado riesgo de 20-29 puntos y alto riesgo $>30$ puntos $[4,12,13]$. Adicionalmente se tomó información de edad, género, tiempo de hospitalización, diagnóstico de ingreso y si falleció o no al egreso de UCIP. Los datos se extrajeron de la base de UCIP y fueron completados con las historias clínicas de cada uno de los casos.

Los datos fueron analizados con el programa estadístico SPSS v20.0. Los datos fueron expresados en frecuencias relativas para variables categóricas y media y desviación estándar para variables numéricas. El poder de discriminación de la prueba, habilidad para distinguir entre los pacientes que van a morir o no, 
Tabla 1. Componentes y puntajes de la escala PRISM (Pediatric Risk of Mortality Score).

\begin{tabular}{|c|c|c|c|c|}
\hline \multirow{2}{*}{ Variable } & \multicolumn{3}{|c|}{ Restricciones por edad y rangos } & \multirow{2}{*}{ Puntaje } \\
\hline & Infantes & Niños & Todos & \\
\hline \multirow[t]{5}{*}{ Presión arterial sistólica (mmHg) } & $130-160$ & $150-200$ & & 2 \\
\hline & $55-65$ & $65-75$ & & 2 \\
\hline & $>160$ & $>200$ & & 6 \\
\hline & $40-54$ & $50-64$ & & 6 \\
\hline & $<40$ & $<50$ & & 7 \\
\hline Presión arterial diastólica (mmHg) & & & $>110$ & 6 \\
\hline \multirow[t]{2}{*}{ Frecuencia cardiaca (latidos/min) } & $>160$ & $>150$ & & 4 \\
\hline & $<90$ & $<80$ & & 4 \\
\hline \multirow[t]{3}{*}{ Frecuencia respiratoria (respiraciones/min) } & $61-90$ & $51-70$ & & 1 \\
\hline & $>90$ & $>70$ & & 5 \\
\hline & Apnea & Apnea & & 5 \\
\hline \multirow[t]{2}{*}{$\mathrm{PaO}_{2} / \mathrm{FiO}_{2}$} & & & $200-300$ & 2 \\
\hline & & & $<200$ & 3 \\
\hline \multirow{2}{*}{$\mathrm{PaCO}_{2}(\mathrm{mmHg})$} & & & $51-65$ & 1 \\
\hline & & & $>65$ & 5 \\
\hline Escala de coma de Glasgow & & & $<8$ & 6 \\
\hline \multirow[t]{2}{*}{ Reactividad pupilar } & & & Diferente o dilatada & 4 \\
\hline & & & No reactiva y dilatada & 10 \\
\hline Tiempo protrombina & & & $>1,5$ & 2 \\
\hline Bilirrubina total (mg/dL) & & & $>3,5$ & 6 \\
\hline \multirow[t]{4}{*}{ Potasio (meq/L) } & & & $3,0-3,5$ & 1 \\
\hline & & & $6,5-7,5$ & 1 \\
\hline & & & $<3,0$ & 5 \\
\hline & & & $>7,5$ & 5 \\
\hline \multirow[t]{4}{*}{ Calcio (mg/dL) } & & & $7,0-8,0$ & 2 \\
\hline & & & $12,0-15,0$ & 2 \\
\hline & & & $<7,0$ & 6 \\
\hline & & & $>15,0$ & 6 \\
\hline \multirow[t]{4}{*}{ Glucosa (mg/dL) } & & & $40-60$ & 4 \\
\hline & & & $250-400$ & 4 \\
\hline & & & $<40$ & 8 \\
\hline & & & $>400$ & 8 \\
\hline \multirow[t]{2}{*}{ Bicarbonato (meq/L) } & & & $<16$ & 3 \\
\hline & & & $>32$ & 3 \\
\hline
\end{tabular}


Tabla 2. Características de los pacientes atendidos en la Unidad de Cuidados Intensivos Pediátricos del Instituto Nacional de Salud del Niño, Lima 2012.

\begin{tabular}{lcl}
\multicolumn{1}{c}{ Característica } & $\mathrm{n}$ & $(\%)$ \\
\hline $\begin{array}{l}\text { Género } \\
\text { Varón }\end{array}$ & 206 & $(53,1)$ \\
Mujer & 182 & $(46,9)$ \\
Edad (años)* & 4,2 & $(5,0)$ \\
Diagnóstico de ingreso & & \\
Postoperatorios & 161 & $(41,5)$ \\
Patología respiratoria & 95 & $(24,5)$ \\
Shock séptico & 51 & $(13,1)$ \\
Neurológicos & 22 & $(5,7)$ \\
Enfermedad cardiovascular & 21 & $(5,4)$ \\
Trauma & 11 & $(2,8)$ \\
Otras enfermedades & 27 & $(7,0)$ \\
\hline
\end{tabular}

* Media (desviación estándar)

se calculó a partir del análisis Receiver Operating Characteristic (ROC), calculándose el área bajo la curva $(A B C)$, actuando la mortalidad observada como gold standard. La calibración, exactitud del riesgo esperado de muerte, fue calculado usando el chi2 de Hosmer Lemeshow comparando la mortalidad observada frente a la predicha, un valor $>0,20$ indica un buen ajuste del modelo. Finalmente, se calculó la tasa de mortalidad estandarizada (TME) que se define como producto de la división entre la mortalidad observada y la mortalidad esperada [14].

\section{RESULTADOS}

De la totalidad de pacientes incluidos $(n=388)$ el $53,1 \%$ de los pacientes fueron de sexo masculino y la edad promedio de fue 4,2 (DE: 5,0) años. Los principales diagnósticos fueron problemas postoperatorios (Tabla 2). El tiempo promedio de estancia en la UCIP fue 12,4 días ( $D E: 15,8$ ) y un $72,3 \%$ requirieron ventilación mecánica, con un tiempo promedio de 6,7 días (DE: 8,5 ).

La mortalidad observada fue de $17,3 \%$ y la tasa de mortalidad estandarizada fue de 1,01. Con respecto a la puntuación del PRISM, 91,2\% de los pacientes fue categorizado como de "bajo riesgo", el 7,0\% de "moderado riesgo" y el 1,8\% de "alto riesgo". El puntaje promedio del PRISM fue de 9,8 (DE: 7,0) entre los pacientes que sobrevivieron. Se encontró asociación entre la mortalidad y la categorización según PRISM (Figura 1, p<0,001).

El área bajo la curva para PRISM fue 0,73 (IC 95\%:0,66-0,80) (Figura 2). La Tabla 3 muestra la calibración de PRIMS para los diferentes deciles de riesgo. El valor p para la prueba de Hosmer y Lemeshow fue de 0,652 , por lo que el modelo de predicción de PRISM tiene un buen ajuste.

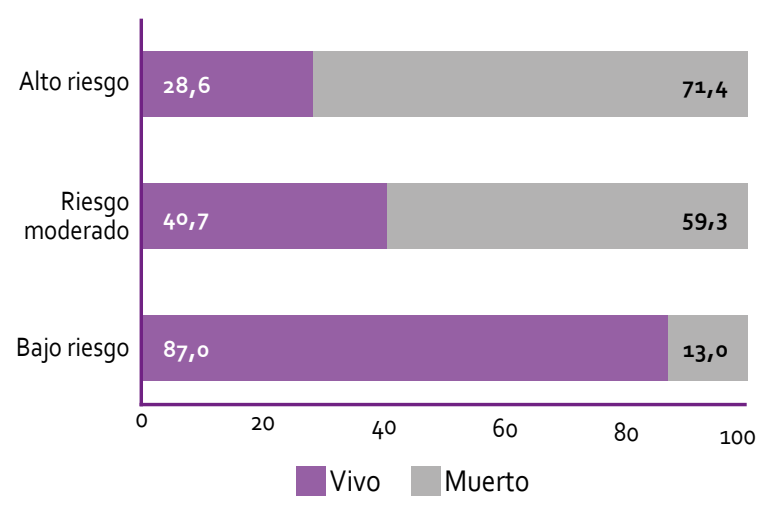

Figura 1. Mortalidad según el score PRISM en pacientes atendidos en la Unidad de Cuidados Intensivos Pediátricos del Instituto Nacional de Salud del Niño, Lima 2012.

\section{DISCUSIÓN}

La aplicación del PRISM, en el presente estudio, ha mostrado ser un instrumento predictor del riesgo de mortalidad al momento del ingreso en los pacientes críticamente enfermos de la UCIP, lo cual está en concordancia con estudios previamente reportados $[2,7,12,13,15-17]$.

Estudios disponibles en la literatura que han evaluado el rendimiento pronóstico del PRISM mediante curvas ROC han mostrado valores de área bajo la curva de 0,73 (IC95\%: 0,66-0,80), 0,85 (IC95\%: 0,79-0,91), y 0,94 (IC95\%: 0,92-

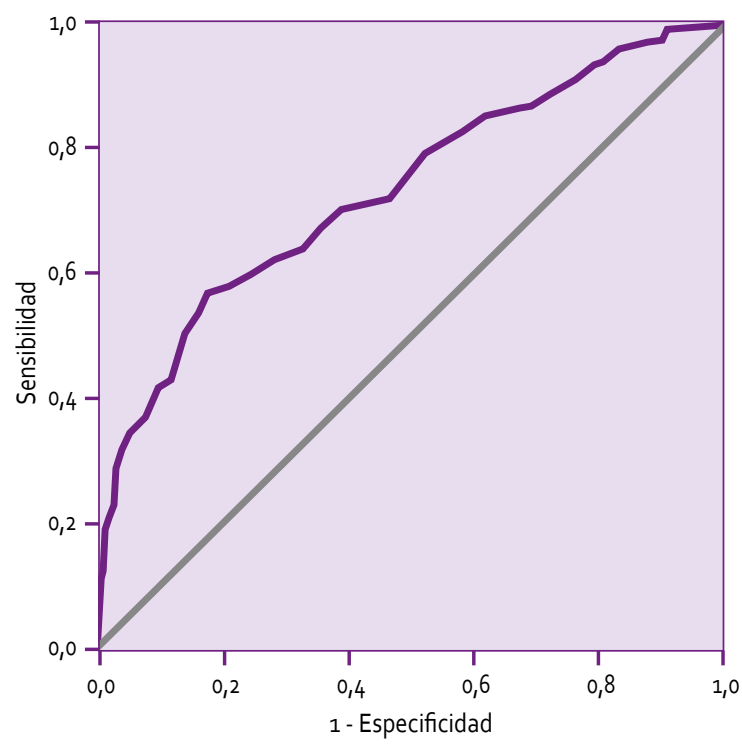

Figura 2. Gráfica del área bajo la curva de respuesta relativa (ROC) para el Score PRISM en paciente pediátrico atendido en la Unidad de Cuidados Intensivos Pediátricos del Instituto Nacional de Salud del Niño, Lima 2012. 
Tabla 3. Calibración del score PRISM, comparando la mortalidad y la supervivencia observada con la esperada en la Unidad de Cuidados Intensivos Pediátricos del Instituto Nacional de Salud del Niño, Lima 2012*.

\begin{tabular}{ccccccc}
\multirow{2}{*}{ Deciles de riesgo } & \multicolumn{2}{c}{ Fallecidos $(\mathrm{n}=67)$} & & \multicolumn{2}{c}{ Sobrevivientes $(\mathrm{n}=\mathbf{3 2 1})$} & Total \\
\cline { 2 - 3 } & Observado & Esperado & & Observado & Esperado & (n=388) \\
\hline 1 & 23 & 21,3 & & 18 & 19,7 & 41 \\
2 & 10 & 10,2 & & 26 & 25,8 & 336 \\
3 & 6 & 6,6 & & 25 & 24,4 & 31 \\
4 & 4 & 7,0 & & 37 & 34,0 & 41 \\
5 & 5 & 6,6 & & 43 & 41,4 & 48 \\
6 & 5 & 2,8 & & 19 & 21,2 & 24 \\
7 & 3 & 3,7 & & 32 & 32,3 & 36 \\
8 & 4 & 3,6 & & 39 & 38,4 & 42 \\
9 & 3 & 2,2 & & 27 & 28,8 & 31 \\
10 & 3,0 & & 55 & 55,0 & 58 \\
\hline
\end{tabular}

* Hosmer Lemeshow $p=0,652$

$0,96)$ en modelos con buena calibración según la prueba de Hosmer y Lemeshow. El rendimiento observado en nuestro estudio fue adecuado $(A B C=0,73)[11]$, el cual si bien fue estadísticamente significativo resultó siendo inferior al valor registrado en estudios anteriores. Sospechamos que uno de los componentes que influye en estos resultados son las características particulares de nuestros pacientes, principalmente la comorbilidad.

Las patologías de los pacientes en la UCIP son variadas. Por ejemplo, en el estudio de Canonero fueron patología respiratoria $(24,7 \%)$, neurología $(9,8 \%)$, los traumatismos $(7,2 \%)$ entre otros. Diferentes resultados comparte Guigñan quien reportó que los diagnósticos de ingreso más frecuentes fueron los cuadros sépticos $(41,2 \%)$, de los cuales la sepsis de cualquier punto de partida representó el $16 \%$, el shock séptico $13,4 \%$ y la sepsis severa $11,8 \%$, y de las patologías no infecciosas el trauma representó el $22 \%$.

La mortalidad en estudios previos varían de $6,6 \%$ a $24,3 \%$. $[7,8,13,15,16]$. La observada en nuestra UCIP está dentro de este rango.

El PRISM aplicado en los pacientes ingresados a la UCIP, presentó correlación con el pronóstico de condición al alta del paciente. Guigñan estima que el mayor porcentaje de mortalidad se ubicó en la categoría de PRISM >31 (83,3\%), resultados similares a los que hemos encontrado en la UCIP del INSN.

En el estudio de Ali Khajeh [18], encontró que el riesgo de mortalidad era 7,2 veces más en los pacientes con puntaje 2130 en comparación con la puntuación de 0-10. El análisis de la curva ROC para la mortalidad según la PRISM mostró un área de la curva de 0,80 . Ellos muestran resultados congruentes con el nuestro y concluyen que PRISM es un buen predictor para la evaluación del riesgo de mortalidad en $\mathrm{UCl}$.

La escala PRISM mostró estar relacionada con la mortalidad observada y la esperada en todos los rangos en la población pediátrica ingresada en la UCIP. EI PRIMS IV el cual limita el periodo de recolección de datos del score a las 4 primeras horas de estancia en $\mathrm{UCl}$, mejora la recolección de información y reduce la posibilidad de sesgo, el nuevo score aparentemente tiene un excelente rendimiento de predicción de mortalidad [19].

El estudio presenta ciertas limitaciones, primero que al ser retrospectivo no se pudo controlar que todas las mediciones se hicieron en las primeras horas de ingreso a UCIP; sin embargo, PRISM considera las primeras 24 horas de ingreso a UCIP. No se calculó el performance del PRISM por tipo de patología de ingreso por tamaño muestral insuficiente, y si bien estos resultados no pueden extrapolarse a otros UCIP peruanas, sin embargo, la UCIP del INSN es la más grande del Ministerio de Salud del Perú.

En conclusión, la aplicación de la escala PRISM muestra un adecuado rendimiento pronóstico y un buen ajuste para la mortalidad en los pacientes hospitalizados en la UCIP del INSN.

Conflicto de interés: Los autores declaran no tener conflictos de interés.

Fuente de financiamiento: Autofinanciado 


\section{REFERENCIAS BIBLIOGRÁFICAS}

1. Epstein $D$, Brill JE. A history of pediatric critical care medicine. Pediatr Res. 2005i58(5):987-96.

2. Gemke RJBJ, Bonsel GJ, van Vught AJ. Outcome assessment and quality assurance in pediatric intensive care. En:Tibboel $D$, van der Voort E. Update in Intensive Care and Emergency Medicine. Berlin: Springer-Verlag; 1996.

3. Gunning K, Rowan K. ABC of intensive care: outcome data and scoring systems. BMJ 1999;319(7204):241-4.

4. Eulmesekian $P$, Pérez $A$, Minces $P$, Ferrero $H$, Fiori T. Validación de dos modelos de predicción de mortalidad, PRISM y PIM2, en una Unidad de Cuidados Intensivos Pediátricos. Arch Argent Pediatr. 2006;104(5):387-92

5. Garcia de Lorenzo A. Scores pronósticos y criterios diagnósticos en el paciente crítico. $2^{\circ}$ edición. Madrid: Ediciones Ergon; 2006.

6. Shann F, Pearson G, Slater A, Wilkinson K. Paediatric index of mortality (PIM): A mortality prediction model for children in intensive care. Intensive Care Med. 1997;23(2):201-7.

7. Singhal D, Kumar N, Puliyel JM, Singh SK, Srinivas V. Prediction of mortality by application of prism score in intensive care unit. Indian Pediatr. 2001;38(7):714-9.

8. Bellad R, Rao S, Patil VD, Mahantshetti NS. Outcome of intensive care unit patients using Pediatric Risk of Mortality (PRISM) score. Indian Pediatr. 2009;46(12):1091-2.

9. Balakrishnan G, Aitchison T, Hallworth D, Morton NS. Prospective evaluation of the Paediatric Risk of Mortality (PRISM) score. Arch Dis Child. 1992;67(2):196-200

10. Wells M, Riera-Fanego JF, Luyt DK, Dance M, Lipman J. Poor discriminatory performance of the Pediatric Risk of Mortality
(PRISM) score in a South African intensive care unit. Crit Care Med. 1996;24(9):1507-13.

11. Pollack MM, Ruttimann UE, Getson PR. Pediatric risks of mortality (PRISM) score. Crit Care Med. 1988;16(11):1110-6.

12. Guigñan $O$, Centritto $C$, Arias $F$, Reyes A. Aplicación de la escala de riesgo de mortalidad pediátrica (PRISM) en una Unidad de Terapia Intensiva Pediátrica Venezolana. Arch Venez Puer Ped. 2007;70(4):126-9.

13. Canonero I, Figueroa A, Cacciamano A, Oliver E, Cuestas E. Validación de los puntajes de mortalidad PRISM y PIMz en una Unidad de Cuidados Intensivos Pediátricos de Córdoba. ArchArgent Pediatr. 2010;108(5):427-33.

14. La Estandarización: un método epidemiológico clásico para la comparación de tasas. Boletín Epidemiológico / OPS. 2002;23(3):9-12.

15. Taori RN, Lahiri KR, Tullu MS. Performance of PRISM (Pediatric Risk of Mortality) Score and PIM (Pediatric Index of Mortality) Score in a Tertiary Care Pediatric ICU. Indian J Pediatr. 2010;77(3):267-71.

16. León R, Tantaleán J, Santos A. Uso del PRISM en una Unidad de Cuidados Intensivos Pediátrica. Intensivos. 2001;3:22-7.

17. Tantaleán J, Paredes L, Santos A, Becerra R. Riesgo de muerte en la unidad de cuidados intensivos pediátricos: Uso del Prism. Rev Peru Pediatr. 2008;61(1):1-8.

18. Khajeh A, Noori NM, Reisi M, Fayyazi A, Mohammadi M, MiriAliabad G. Mortality risk prediction by application of pediatric risk of mortality scoring system in pediatric intensive care unit. Iran J Pediatr. 2013;23(5):546-50.

19. Pollack MM, Holubkov R, Funai T, Dean JM, Berger JT, Wessel DL, et al The Pediatric Risk of Mortality Score: Update 2015. Pediatr Crit Care Med. 2016;17(1):2-9.

\section{Las ediciones anteriores de Acta Médica Peruana están disponibles en:}

\section{www.redalyc.org}

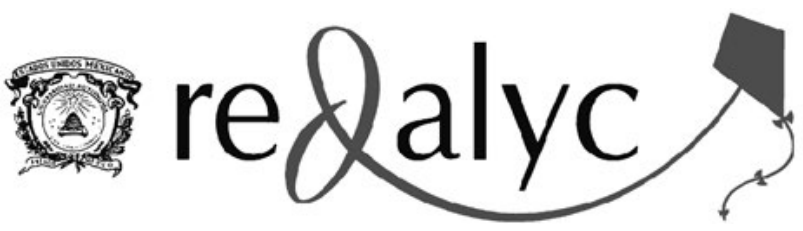

